\title{
In Vitro Comparison of Two Widely Used Surgical Sealants for Treating Alveolar Air Leak
}

\author{
Ruoyu Zhang ${ }^{1, *}$ Maximilian Bures ${ }^{1, *}$ Klaus Höffler $^{1}$ Danny Jonigk ${ }^{2}$ Axel Haverich ${ }^{1}$ Marcus Krueger ${ }^{1}$ \\ ${ }^{1}$ Department of Cardiac, Thoracic, Transplantation and Vascular \\ Surgery, Hannover Medical School, Hannover, Germany \\ 2 Department of Pathology, Hannover Medical School, Hannover, \\ Germany \\ Address for correspondence Ruoyu Zhang, MD, Department of \\ Cardiac, Thoracic, Transplantation and Vascular Surgery, Hannover \\ Medical School, Carl-Neuberg-Str. 1, Hannover 30625, Germany \\ (e-mail: zhang.ruoyu@mhhannover.de).
}

Thorac Cardiovasc Surg 2014;62:e1.

\section{ERRATUM}

Both authors contributed equally to this work.
It has been brought to the Editor's attention that the above mentioned manuscript was written with the two first authors as equally contributing ones and should therefore have been marked accordingly. The article was published in the issue "Thorac Cardiovasc Surg 2014;62(8):705-709." DOI: 10.1055/s-0034-1389272.

The authors' respective contributions are as follows:

\section{Dr. med. Ruoyu Zhang}

Dr. Zhang carried out the theoretic conception of the project as well as the conception of the study design. He participated in the creation of the experimental protocol and the conduction of the experiments. Dr. Zhang's assignments further include the evaluation and interpretation of the acquired data including graphic analysis. He drafted the paper with special regards to background, discussion and conclusion. Overall, the work on this project was characterized by very close cooperation. Throughout the writing process, all content was subject to constant bilateral revision with Mr. Maximilian Bures. Dr. Zhang was the corresponding author and attests that Mr. Bures was considered to be an equally contributing first author from the outset of the project.

\section{Maximilian Bures}

Mr. Bures participated in the creation of the experimental protocol. He aided in the conception of the study design and led the conduction of the experiments, while further improving the study concept. His assignments further encompassed the evaluation and interpretation of the acquired data, including graphic analysis. He drafted the paper with special regards to the sections materials \& methods, results and discussion. The work on this project was characterized by very close cooperation. Throughout the writing process, all content was subject to constant bilateral revision with Dr. Ruoyu Zhang.

Accordingly, the authorships are changed and correct authorships appear as above. received

November 24, 2016 accepted after revision

November 25, 2016 (c) 2014 Georg Thieme Verlag KG Stuttgart · New York
DOI http://dx.doi.org/ 10.1055/s-0036-1597906. ISSN 0171-6425. 